\title{
Development of a RAPD-Based Male-Specific Molecular Marker in Japanese HoP (Humulus JAPONicus Siebold \& Zucc.)
}

\author{
Patryk Mizia ${ }^{1}$, Magdalena Cygan ${ }^{1 *}$, Magdalena Denysenko ${ }^{1}$, \\ DAGMARA KWOLEK ${ }^{1}$, ANDRZEJ CHRAMIEC-GŁĄiK ${ }^{1}$, \\ AlEKSANDRA GRABOWSKA-JOACHIMIAK ${ }^{2}$ AND ANDRZEJ J. JOACHIMIAK ${ }^{1}$ \\ ${ }^{1}$ Department of Plant Cytology and Embryology, Institute of Botany, \\ Jagiellonian University, Gronostajowa 9, 30-387 Cracow, Poland \\ ${ }^{2}$ Department of Plant Breeding and Seed Science, University of Agriculture in Cracow, \\ Łobzowska 24, 31-140 Cracow, Poland
}

Received September 7, 2016; revision accepted September 28, 2016

\begin{abstract}
The male-specific DNA markers are very useful in molecular sexing of non-flowering plants and seeds of dioecious species. In this paper we identified ten Y chromosome-specific RAPD primers suitable for identification of male plants in three Cannabaceae species with sex chromosomes (Humulus lupulus, XX/XY; H. japonicus, XX/ XY1Y2; Cannabis sativa, XX/XY). Basing on the nucleotide sequence of the OPJ-09 RAPD product we developed the HJY09 SCAR marker, which is very efficient in sexing of Japanese hop.
\end{abstract}

Keywords: Cannabaceae, Humulus japonicus, SCAR marker, plant sexing

\section{INTRODUCTION}

The Japanese hop (Humulus japonicus Siebold \& Zucc.) is an annual species of the small family Cannabaceae, which is the only angiosperm family consisting exclusively of dioecious species with heteromorphic sex chromosomes. Unlike its closest relative, the common hop (Humulus lupulus), the Japanese hop is not useful for brewing industry, due to lack of lupulin glands. In recent years, extracts from $H$. japonicus have been proven to possess various antioxidative, anti-aging, antibacterial, antimutagenic, anti-inflammatory and antitumor properties (Sung et al., 2015).

$H$. japonicus is characterized by a multiple (polymorphic) sex chromosome system $(2 \mathrm{n}=16, \mathrm{XX}$ in females and $2 \mathrm{n}=17, \mathrm{XY} 1 \mathrm{Y} 2$ in males) (Sinoto, 1929; Winge, 1929) which distinguishes it from two other Cannabaceae species, the common hop (H. lupulus) and hemp (Cannabis sativa), both possessing $2 \mathrm{n}=20$ chromosomes and the simple $\mathrm{XX} / \mathrm{XY}$ chromosome system (Vyskot and Hobza, 2004). The lower chromosome count and different sex chromosome system suggest that $H$. japonicus has derived and highly reorganized karyotype with a sex chromosome system originating from the $\mathrm{XX} /$ $\mathrm{XY}$ one. Regardless of the sex chromosome system, sex determination in Cannabaceae relies on $\mathrm{X}$-to-autosome balance $(\mathrm{X} / \mathrm{A} \geq 1.0$ in females and $\mathrm{X} / \mathrm{A} \leq 0.5$ in males) (Ainsworth, 2000). In terms of both sex chromosome and sex determination systems, H. japonicus resembles Rumex acetosa, a model species in sex chromosome research in plants (Navajas-Perez, 2012). Despite the clear karyotypic difference between them, both hop species showed almost identical cpDNA organization. Moreover, comparative analysis of nuclear rDNA coding regions in $H$. lupulus, $H$. japonicus and C. sativa revealed high similarity of Cannabaceae species in this respect (Pillay and Kenny, 2006).

The two species with a simple sex chromosome system (C. sativa and $H$. lupulus) differ drastically in the size of the Y chromosome. Moreover, H. lupulus is the only angiosperm species with the $\mathrm{Y}$ much smaller than X. It may arouse suspicion that sex chromosomes observed in different Cannabaceae

\footnotetext{
* Corresponding author, email: cyganka90@gmail.com
} 
species might not show common origin. In animals there is a well-known and frequently described phenomenon called sex chromosome turnover, which causes that the ancestral sex chromosomes are not conserved during evolution even in closely related species. Sex chromosome turnover is particularly probable in organisms possessing the sex determination system X:A, e.g. in Drosophila (Carvalho and Clark, 2005). Because Y chromosomes in both Humulus species demonstrate significant differences (Y chromosome in $H$. lupulus is completely euchromatic and small, whereas Y chromosomes in $H$. japonicus are big and heterochromatinized), their homology requires investigation.

In comparison to $H$. lupulus and $C$. sativa, which are commercially important species, genetic studies on $H$. japonicus were neglected for a long time. One of the obstacles in genetic research on the Japanese hop is the paucity of molecular markers suitable for studies on the karyotype evolution in this species and useful in identification of sex chromosomes and in molecular sexing of non-flowering plants and seeds. In the last years, however, some progress has been made, mainly in the knowledge of the karyotype structure and genome size in H. japonicus (Grabowska-Joachimiak et al., 2006, 2011; Kim et al., 2008; Alexandrov et al., 2012). More recently, a sex chromosome-specific DNA library has been constructed in this species with the use of laser microdissection (Yakovin et al., 2014).

Grabowska-Joachimiak et al. (2011) suggested that the chromosome complement of $H$. japonicus could have arisen by the X-autosome plus autosome-autosome fusions. Such events have led to the emergence of second Y chromosome and reduction in the autosome number from 18 to 14 . The autosome fusion was confirmed by interstitial telomeric sequences located in the large autosome pair, but molecular traces of the X-autosome fusion were not found. Thus, the origin of $H$. japonicus XX/XY1Y2 sex chromosome system from this presented in $H$. lupulus still needs confirmation. A good way to shed light on this question could be search for male-specific (Y-specific) DNA sequences occurring in both species. The X-autosome fusion retains an original Y in the derived sex chromosome system, and some DNA markers located on this chromosome should be observed in both species.

To date, only two Y chromosome-markers have been developed in the Japanese hop. Aleksandrov et al. (2011) described K-16, a male specific ISSR (Inter Simple Sequence Repeat) marker, while Gao et al. (2010) developed a male specific SCAR (Sequence Characterized Amplified Region) marker SEX I64 which was based on ISSR marker I64. According to our findings, both markers were ineffective in $H$. lupulus (Mizia, unpublished results). In this study, we performed RAPD analysis of
$H$. japonicus plants in order to develop other malespecific markers useful in sexing of plants and future studies of Y chromosomes in hops.

\section{MATERIAL AND METHODS}

\section{PLANT MATERIAL}

Molecular analysis of $H$. japonicus included 31 flowering plants (16 males and 15 females) grown from purchased seeds (W. Legutko plant breeders, Poland). Karyological analysis showed that chromosome constitution of all these plants was consistent with their sex phenotype (males $2 \mathrm{n}=17$ and females $2 n=16$ ). The usefulness of the developed HJYO9 SCAR primers was tested on seeds from Vilmorin Garden plant breeders (France) and PlantiCo plant breeders (Poland). The analyzed Humulus lupulus plants (5 males and 5 females) originated from a wild population in Rękawka (Kraków, Poland). The examined Cannabis sativa plants ( 5 males and 5 females) were obtained from commercially available seeds (PPUH Bratek, Poland).

\section{DNA ISOLATION}

DNA was isolated from fresh leaves using CTAB procedure (Gavel and Jarret, 1991) with the modification described in Mizia et al. (2014). DNA from seeds was isolated using Gene MATRIX FoodExtract DNA purification Kit (EURx Sp. z o.o. Gdansk, Poland).

\section{RAPD REACTIONS}

Thirty RAPD primers were pre-tested on five male and five female $H$. japonicus plants (Tab. 1). Three of them (OPA-07, OPJ-09 and OPU-08) were previously described by Polley et al. (1997) as producing male-specific DNA fragments in $H$. lupulus, whereas two others (RAPD 8 and RAPD 11) were described as producing male-specific DNA fragments in C. sativa (Sakamoto et al., 1995). RAPD reaction mixture and amplification were made as described in Mizia et al. (2014). Only primers generating reproducible band profiles were used in further research.

\section{DNA FRAGMENT ISOLATION, CLONING AND SEQUENCING}

Male specific bands were extracted from $1 \%$ agarose gel with 1X TBE using GenMatrix AgaroseOut DNA Purification Kit (EURx Sp. z o.o. Gdansk, Poland). The purified products were then ligated with an E. coli plasmid (Green and Sambrook, 2012). Ligation was performed using CloneJET PCR Cloning Kit (Thermo Scientific). Plasmids purifications were performed using GeneMATRIX Plasmid 
TABLE 1. Nucleotide sequences of tested random primers; RAPD - Sakamoto et al., 1995, OPA-04 - OPU-08 - Operon Technologies Inc.

\begin{tabular}{|c|c|c|c|}
\hline Primer & Sequence (5'-3') & Primer & Sequence (5'-3') \\
\hline RAPD1* & GCAAGTAGCT & OPA- $04^{*}$ & AATCGGGCTG \\
\hline RAPD2* & TGGCTCAAAG & OPA-07* & GAAACGGGTG \\
\hline RAPD3* & CAGTGTGTGG & OPA-17 & GACCGCTTGT \\
\hline RAPD4* & GTGTCAGGCA & OPB-07 & GGTGACGCAG \\
\hline RAPD5* & ATACCATCCC & OPC- $01^{*}$ & TTCGAGCCAG \\
\hline RAPD6 & GATCCCCTGA & OPC-10 & TGTCTGGGTG \\
\hline RAPD7* & GATAACCGCA & OPD-12 & CACCGTATCC \\
\hline RAPD8* & ATCCGCGTTC & OPD-17 & TTTCCCACGG \\
\hline RAPD9* & CCAGTGGTTC & OPE-05 & TCAGGGAGGT \\
\hline RAPD $10 *$ & TGACGATGCA & OPF-11 & TTGGTACCCC \\
\hline RAPD11* & ACGGCATATG & OPG-20 & TCTCCCTCAG \\
\hline RAPD12* & TAACCATCCC & OPH-14 & ACCAGGTTGG \\
\hline RAPD13* & ATGTCCGCAC & OPI-03 & CAGAAGCCCA \\
\hline RAPD14* & GTGTGGATGG & OPJ-09* & TGAGCCTCAC \\
\hline RAPD15 & GTCGTTACGA & OPU-08* & GGCGAAGGTT \\
\hline
\end{tabular}

* - used in further investigation

Miniprep DNA Purification Kit (EURx Sp. z o.o. Gdansk, Poland). The purified plasmids were then sent to Genomed S.A. (Warsaw, Poland) for sequencing. SCAR primers were designed based on the obtained sequences. All male-specific sequences were uploaded into GenBank and received accession numbers (KX688586- KX688595).

\section{SCAR REACTION MIXTURE AND AMPLIFICATION}

PCR mixture for HJY09 and SCAR OPJ-09 (H. lupulus from Polley et al., 1997) consisted of ( $15 \mu$ total) 1X Thermo Scientific DreamTaq Green Buffer (including $20 \mathrm{mM} \mathrm{MgCl}_{2}$ ); $0.25 \mathrm{mM}$ dNTPs; $0.25 \mu \mathrm{M}$ of each primer; $1.13 \mathrm{U}$ DreamTaq DNA Polymerase (Thermo Scientific) and 60 ng of DNA and was run in Bio-Rad T100 Thermal Cycler. Amplifications were done according to the following program: initial denaturation step at $94^{\circ} \mathrm{C}$ for 4 min.; 30 cycles consisting of denaturation step at $94^{\circ} \mathrm{C}$ for $1 \mathrm{~min}$., primer annealing step at $60^{\circ} \mathrm{C}$ for $45 \mathrm{~s}$ and primer extending step at $72^{\circ} \mathrm{C}$ for $1 \mathrm{~min}$ $30 \mathrm{~s}$; and final extending step at $72^{\circ} \mathrm{C}$ for $8 \mathrm{~min}$. The PCR products were separated in $1 \%$ agarose gel with 1X TBE and SimplySafe (EURx Sp. $z$ o.o. Gdansk, Poland). PCR reaction mixture and ampli- fication for SEX I64 were carried in Bio-Rad T100 Thermal Cycler according to the instructions in Gao et al. (2010).

\section{ANALYSIS OF OBTAINED SEQUENCES}

Sequences of both OPJ-09 male-specific products obtained from $H$. japonicus were compared with sequence of male-specific product generated by OPJ09 for H. lupulus by using MultAlin program (Corpet, 1988). Each comparison was made using default settings (best math) and with a gap penalty at opening and extension. High penalty allowed stretching sequences from forward to reverse primer (Fig. S1, supplementary material).

H. japonicus OPJ-09 700 bp sequence was used in nucleotide BLAST search (Camacho et al., 2009) on megablast and discontiguous megablast settings.

\section{RESULTS AND DISCUSSION}

The development of sex-chromosome markers can be beneficial in obtaining information useful in basic research on sex determination and sex-specific genome regions (Charlesworth, 2008). Such 
markers, located mainly on Y chromosomes, enabled progress in research on sex chromosomes of several dioecious species, including two model species, Silene latifolia (Kazama and Matsunaga, 2008) and Rumex acetosa (Mariotti et al., 2009; NavajasPerez, 2012), as well as commercially important Cannabis sativa (Sakamoto et al., 1995), Humulus lupulus (Divashuk et al., 2011), Carica papaya (Liu et al., 2004) and Asparagus officinalis (Gao et al., 2007). They were also used for sexing of unflowering plants or seeds (Polley et al., 1997; Zhang et al., 1998; Korpelainen, 2002; Danilova and Karlov, 2006; Gangopadhyay et al., 2007; Hobza and Widmer, 2008; Kwolek and Joachimiak, 2011). The PCR-based DNA fingerprinting techniques commonly used in the development of sex-linked markers in plants include, among others, RAPD (Random Amplified Polymorphic DNA), in which short (usually $10 \mathrm{bp}$ ) arbitrary primers are used to generate random genomic fragments, as well as ISSR (Inter Simple Sequence Repeat), which is based on amplification of genome regions between neighboring and inversely oriented microsatellites. The obtained DNA fragments are sometimes used to generate sequence specific markers (STS, SCAR) which are more advantageous (Heikrujam et al., 2015), because they show high reproducibility and are locus-specific.

From the 30 RAPD primers tested in this research, only 18 yielded reproducible band profiles in $H$. japonicus plants. Ten out of these primers also generated male-specific DNA fragments in particular Cannabaceae species (Tab. 2). Only two primers (OPU-08 and OPJ-09) produced male-specific bands both in $H$. japonicus and $H$. lupulus. The male-specific primer common for Humulus and Cannabis was not found. RAPD profiles produced by OPJ-09 primer showed one male-spe-

TABLE 2. RAPD primers producing male-specific products (MSP) in Humulus japonicus, H. lupulus and Cannabis sativa

\begin{tabular}{|c|c|c|c|c|c|c|}
\hline \multirow[t]{2}{*}{ Primer } & \multicolumn{2}{|c|}{ H. japonicus } & \multicolumn{2}{|c|}{ H. lupulus } & \multicolumn{2}{|c|}{ C. sativa } \\
\hline & Number of MSP & $\begin{array}{l}\text { Length of MSP } \\
\text { [bp] }\end{array}$ & Number of MSP & Length of MSP [bp] & Number of MSP & $\begin{array}{l}\text { Length of MSP } \\
\text { [bp] }\end{array}$ \\
\hline OPA-07 & 0 & - & 1 & 1700\# & 0 & - \\
\hline OPC-01 & 1 & $\begin{array}{c}450^{*} \\
(\mathrm{KX} 688586)\end{array}$ & 0 & - & NT & - \\
\hline OPJ-09 & 2 & $\begin{array}{c}1600^{*} \\
(\mathrm{KX688590)} \\
700^{*} \\
(\mathrm{KX} 688591)\end{array}$ & 1 & $\begin{array}{c}\text { 1200\#* } \\
(\mathrm{KX688593)}\end{array}$ & 0 & - \\
\hline OPU-08 & 1 & $\begin{array}{c}900^{*} \\
(\mathrm{KX} 688592)\end{array}$ & 1 & $1400 \#$ & 0 & - \\
\hline RAPD 2 & 1 & $\begin{array}{c}2000^{*} \\
\text { (KX688594 - } \\
\text { partial } \\
\text { KX688595 - } \\
\text { partial) }\end{array}$ & 0 & - & 0 & - \\
\hline RAPD 3 & 1 & $\begin{array}{c}300^{*} \\
(\mathrm{KX688587)}\end{array}$ & 0 & - & 0 & - \\
\hline RAPD 5 & 2 & $\begin{array}{c}1200 \\
100 *^{*} \\
(\mathrm{KX} 688589)\end{array}$ & 0 & - & 0 & - \\
\hline RAPD 8 & 0 & - & NT & - & 1 & $500^{\$}$ \\
\hline RAPD 11 & 0 & - & NT & - & 1 & $730^{\$}$ \\
\hline RAPD 13 & 3 & $\begin{array}{c}1100 \\
800^{*} \\
(\mathrm{KX} 688588) \\
900\end{array}$ & 0 & - & 0 & - \\
\hline
\end{tabular}

* - sequenced by us; \# - sequenced by Pooley et al. (1997); \$ - sequenced by Sakamoto et al. (1995); grey - products used to obtain efficient male-specific STS/SCAR markers. NT -not tested 
cific fragment in $H$. lupulus $(\sim 1.2 \mathrm{~kb})$ and two in H. japonicus ( $\sim 1.6 \mathrm{~kb}$ and $\sim 700 \mathrm{bp}$ ) (Fig. 1). OPU08 generated one male-specific product both in $H$. lupulus and $H$. japonicus. The product obtained in $H$. japonicus $(\sim 1 \mathrm{~kb})$ was smaller than the one obtained in $H$. lupulus $(\sim 1.4 \mathrm{~kb})$.

Eight out of eleven male-specific $H$. japonicus RAPD products were cloned and sequenced, and respective SCAR primers were designed. Testing reactions with these primers showed that only one marker (HJY09, based on the shorter OPJ-09 fragment) was highly male-specific in $H$. japonicus plants. The starting OPJ-09 sequence used for the generation of HJY09 marker is given in the Fig. 3. The suitability of our SCAR marker in sexing of $H$. japonicus was also tested on DNA isolated from seeds. The efficiency of this marker in identification of male seeds was confirmed by using the Y-specific SEX I64 marker developed by Gao et al. (2010) (Fig. 2). Unfortunately, HJYO9 primers designed by us (5'-TCACCACTTTGAACTCGCTG-3' and 5'-CCTTGTCTGGGTCGATTTGT-3') proved to be inef- fective in $H$. lupulus males (data not shown). BLAST analysis of OPJ09 700 bp product did not shown any significant similarity to any sequence stored in database.

In angiosperms, the occurrence of the multiple XX/XY1Y2 sex chromosome system was documented only in some species of Rumex (representatives of European section Acetosa and in American $R$. hastatulus) and in $H$. japonicus (Mariotti et al., 2009; Grabowska-Joachimiak et al., 2011, 2015). According to the most common hypothesis, the polymorphic sex chromosome system derives from the simple ones (XY) by fusion between an autosome and a sex chromosome (Ohno, 1967; White, 1973). If so, the derived $X X / X Y 1 Y 2$ system of $H$. japonicus should possess both an ancestral and a neo-Y chromosome (of autosomal provenience). The occurrence of male-specific markers amplified by the same primers in the two Humulus species could confirm this supposition.

In this study we focused primarily on finding reproducible, sequence-specific sex markers

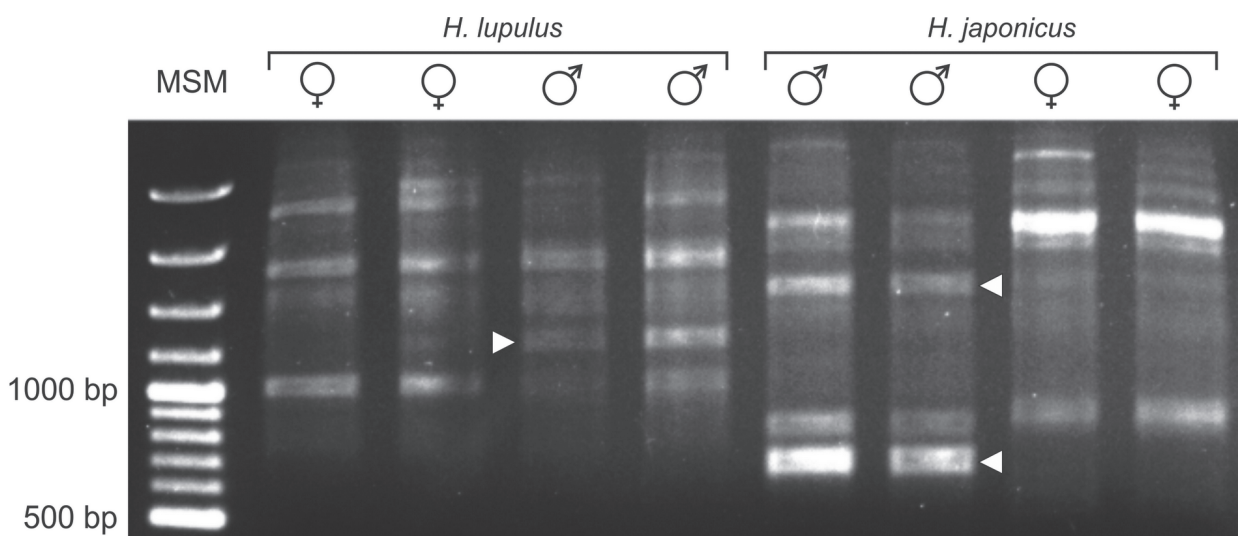

Fig. 1. RAPD profile obtained using OPJ-09 RAPD primer in Humulus lupulus and H. japonicus. MSM - molecular size marker (100 bp DNA ladder plus - Thermo Scientific); Male-specific fragments marked by arrowheads.

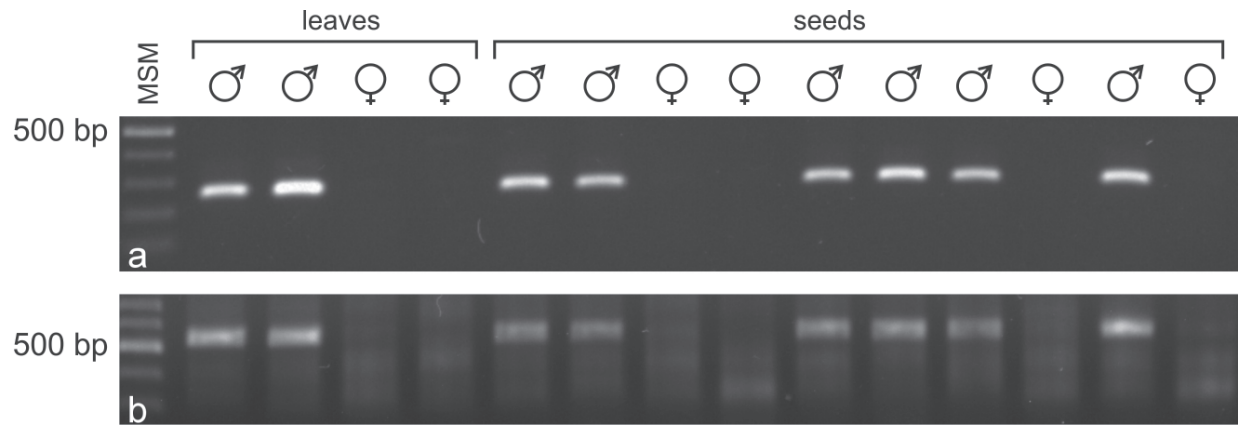

Fig. 2. DNA fragments produced for Humulus japonicus plants and seeds by two male-specific markers based on OPJ-09 product: (a) HJY09, (b) SEX I64. MSM - molecular size marker (100 bp DNA ladder plus - Thermo Scientific). 
TGAGCCTCACCACTTTGAACTCGCTGAATGTTATTAATTGCTGTCCCATGTTATTGAAGA GATGCAAAAGGGAAATAGGTGAAGATTGGGATAAGATTTCTCATATACAACACTTACTCT TACGTCCAGATCCCAACGAAAAAGAGGATGAGGTAGAAAATTTAACTTAATCATTTTATT TTTATTAAATATAACCTAAGTGCTCAGTTTGATTTAGTTTAATTTATTTAACACTGCAGAAT GCGTTGGAAGAGGAAACAAATCGACCCAGACAAGGGGATGCAACCTCTTGTTGAACAA GTTTAGGGTATGCAATATTATGAGTACAAGAATTCCAAAATAGATATGTGCAGGTAATTA TCCTGCTAATCCCTTCTACCTGTATAAACTTCATTCTAATATGATTTAAATGACAAATCTC AATCTAAAATAAGAACACTTTTCAGCCATTTGAAAGAAAAATATATTTTATCTGTTCAATTA AAAATCTTGAGCTTATGATTCGTTTGTAAGAACTTCTTACTTTTCTTTTGAGTTCTTGAAA GACTAAAGCATATCAAAATCCCATTTTGTGGTGATAACATGTTGGTAATATAGGGGGCAT GCTATGGGTTGAAATTACATTAATGTTCCACTTGGGACTGACTGGTCTGCTACACCTGGT TATAAAATCTGGTCATCGAGTGAGGCTC

Fig. 3. Nucleotide sequence of smaller male-specific product generated by OPJ-09 (692 bp). Underlined - the sites of designed HJY09 SCAR primers; Grey - 270 bp fragment produced by designed primers.

in $H$. japonicus, but we also aimed to find RAPD primers effective in generating Y-specific DNA fragments in two Humulus species. We found two RAPD primers (OPJ-09 and OPU-08) producing Y-specific bands both in $H$. japonicus and $H$. lupulus. The same primers, tested by Törjék et al. (2002) in Cannabis sativa, gave no sex-specific products in this species. 1200 bp DNA fragment amplified by OPJ-09 in $H$. lupulus was used by Polley et al. (1997) to design the Y-specific STS primers for this species. We tested these primers (5'-ACAGAGTACAACTCAGAAACAAACC-3' and 5'-AAGGTCGCACAATGACCG-3') in $H$. japonicus, but they did not amplify any DNA fragments, neither in males nor in females (data not shown). Because the nucleotide sequence of OPJ-09 product in $H$. lupulus has not been published by authors, we cloned and sequenced this DNA fragment. It turned out that the similarity of the OPJ-09 products generated in two Humulus species was too small to conclude their common origin (see Fig. S1 in supplementary material). Thus, the occurrence of common OPJ-09 RAPD markers does not prove that $H$. japonicus sex chromosome system originated from the X-autosome fusion in the common ancestor of the two analyzed species. Further molecular-cytogenetic studies with the use of the Y-specific DNA sequences should clarify this issue.

\section{AUTHORS’ CONTRIBUTION}

AJJ and AG-J plant material, original idea, study design, karyological analysis and critical revision of manuscript; DK, PM, AC-G, MC, MD acquisition of data; DK, PM analysis and interpretation of data; AC-G and PM drafting of manuscript. The authors declare that there are no conflicts of interests.

\section{ACKNOWLEDGEMENTS}

This study was supported by the Department of Plant Cytology and Embryology, Jagiellonian University (subvention No DS/MND/WBiNoZ/ IB/14/2013 and DS/MND/WBiNoZ/IB/8/2015).

\section{REFERENCES}

Ainsworth C. 2000. Boys and girls come out to play: the molecular biology of dioecious plants. Annals of Botany 86(2): 211-221. DOI:10.1006/anbo.2000.1201

Aleksandrov OS, Divashuk MG, and Karlov GI. 2011. Development of a sex-specific molecular marker for Japanese hop Humulus japonicus Siebold \& Zucc. Russian Journal of Genetics 47(8): 1016-1020. DOI:10.1134/ S1022795411080023

Alexandrov OS, DivashuK MG, YaKovin NA, and Karlov GI. 2012. Sex chromosome differentiation in Humulus japonicus Siebold \& Zuccarini, 1846 (Cannabaceae) revealed by fluorescence in situ hybridization of subtelomeric repeat. Comparative Cytogenetics 6(3): 239-247. DOI: 10.3897/CompCytogen.v6i3.3261

Camacho C, Coulouris G, Avagyan V, Ma N, Papadopoulos J, BEALER K and MADDEN TL. 2009. BLAST+: architecture and applications. BioMed Central Bioinformatics 10: 421. DOI: $10.1186 / 1471-2105-10-421$

CARVAlHo AB, and Clark AG. 2005. Y chromosome of D. pseudoobscura is not homologous to the ancestral Drosophila Y. Science 307: 108-110.

CHARlesworth D. 2008. Plant sex chromosomes. In: Volff J-N (ed), Plant Genomes, Genome Dynamics, 83-94. Karger, Basel.

CORPET F. 1988. Multiple sequence alignment with hierarchical clustering. Nucleic Acids Research 16(22): 1088110890. DOI: $10.1093 /$ nar/16.22.10881

DANILOVA TV, and Karlov GI. 2006. Application of Inter Simple Sequence Repeat (ISSR) polymorphism for detection of sex-specific molecular markers in Hop (Humulus lupulus L.). Euphytica 151: 15-21. 
Divashuk MG, Alexandrov OS, Kroupin Y, and Karlov GI. 2011. Molecular cytogenetic mapping of Humulus lupulus sex chromosomes. Cytogenetic and Genome Research 134: 213-219. DOI: $10.1159 / 000328831$

GaO WJ, Li RL, Li SF, Deng CL, and Li SP. 2007. Identification of two markers linked to the sex locus in dioecious Asparagus officinalis plants. Russian Journal of Plant Physiology 54: 816-821.

GaO WJ, Sha T, Ji YK, Deng CL, and Lu LD. 2010. Clone and development of ISSR and SCAR markers linked to male Humulus scandens. Journal of Tropical and Subtropical Botany 18: 283-287.

Gangopadhyay G, Roy SK, Ghose K, Poddar R, Bandyopadhyay T, Basu D, and MukherJee KK. 2007. Sex detection of Carica papaya and Cycascircinalis in preflowering stage by ISSR and RAPD. Current Science 92: 524-526.

GAVEL NJ, and JARRET RL. 1991. A modified CTAB DNA extraction procedure for Musa and Ipomoea. Plant Mololecular Biology Reporter 9: 262-266.

Grabowska-Joachimiak A, Sliwinska E, PiguŁa M, Skomra U, and JOACHIMIAK AJ. 2006. Genome size in Humulus lupulus L. and H. japonicus Siebold \& Zucc. (Cannabaceae). Acta Societatis Botanicorum Poloniae 75: 207-214.

Grabowska-JoachimiaK A, MosioleK M, Lech A, and GóralSKI G. 2011. C-banding/DAPI and in situ hybridization reflect karyotype structure and sex chromosome differentiation in Humulus japonicus Siebold \& Zucc. Cytogenetic and Genome Research 132(3): 203-211. DOI: $10.1159 / 000321584$

GrabowsKa-Joachimiak A, Kula A, Ksiaż̇zyk T, ChOJNicka J, SLIWINSKA E, and JOACHIMIAK AJ. 2015. Chromosome landmarks and autosome-sex chromosome translocations in Rumex hastatulus, a plant with XX/XY1Y2 sex chromosome system. Chromosome Research 23(2): 187-197. DOI: $10.1007 / \mathrm{s} 10577-014-9446-4$

Green MR, AND SAMBrook J. 2012. Molecular Cloning a Laboratory Manual. Cold Spring Harbor, New York.

Heikrujam M, Sharma K, Prasad M, and Agrawal V. 2015. Review on different mechanisms of sex determination and sex-linked molecular markers in dioecious crops: a current update. Euphytica 201(2): 161-194. DOI:10.1007/ s10681-014-1293-z

Hobza R, and Widmer A. 2008. Efficient molecular sexing in dioecious Silene latifolia and S. dioica and paternity analysis in F1 hybrids. Molecular Ecology Resources 8(6): 1274-6.

Kazama Y, and Matsunaga S. 2008. The use of repetitive DNA in cytogenetic studies of plant sex chromosomes. Cytogenetic and Genome Research 120: 247-254. DOI: 10.1159/000121074

KIM S-Y, KIM C-S, LEE J, and BANG J-W. 2008. Karyotype analysis and physical mapping using two rRNA genes in dioecious plant, Humulus japonicus Siebold \& Zucc. Genes and Genomics 30: 243-251.

KoRPELAINEN H. 2002. A genetic method to resolve gender complements investigations on sex ratios in Rumex acetosa. Molecular Ecology 11: 2151-2156.

KwoleK D, and JOACHIMIAK AJ. 2011. Seed sexing revealed female bias in two Rumex species. Acta Societatis Botanicorum Poloniae 80: 93-97.
Liu Z, Moore PH, Ma H, Ackerman CM, Ragiba M, Yu Q, Pearl HM, Kim MS, Charlton JW, Stiles JI, Zee FT, Paterson AH, and Ming R. 2004. A primitive Y chromosome in papaya marks incipient sex chromosome evolution. Nature 427: 348-352.

Mariotti B, Manzano S, Kejnovsky E, Vyskot B, and JamileNA M. 2009. Accumulation of Y-specific satellite DNAs during the evolution of Rumex acetosa sex chromosomes. Molecular Genetics and Genomics 281: 249-259. DOI: $10.1007 / \mathrm{s} 00438-008-0405-7$

MizIAP, KWOLEKD, and ILNICKI T. 2014. DNA stability contrasts with chromosome variability in Allium fistulosum calli. Acta Biologica Cracoviensia Series Botanica 56(1): 66-72. DOI: 10.2478/abcsb-2014-0005

NAVAJAS-PÉrez R. 2012. The genus Rumex: A plant model to study sex-chromosome evolution. In: Navajas-Pérez R, ed. New insights on plant sex chromosomes, 91-109. Nova Science Publishers Inc., New York.

Oнnо S. 1967. Sex chromosomes and sex-linked genes. Springer-Verlag, Berlin.

Pillay M, and Kenny ST. 2006. Structural organization of the nuclear ribosomal RNA genes in Cannabis and Humulus (Cannabaceae). Plant Systematics and Evolution 258: 97-105.

Polley A, Seigner E, and Ganal MW. 1997. Identification of sex in hop (Humulus lupulus) using molecular markers. Genome 40: 375-361.

SaKamoto K, Shimomura K, Komeda Y, Kamada H, and Satoh S. 1995. A male-associated DNA sequence in dioecious plant, Cannabis sativa L. Plant and Cell Physiology 36(8): 1549-1554.

Sinoto Y. 1929. Chromosome studies in some dioecious plants with special reference to the allosomes. Cytologia 1: 109-191.

Sung B, Chung J, BAe H, ChOI J, Kim C, and KIm N. 2015. Humulus japonicus extract exhibits antioxidative and antiaging effects via modulation of the AMPK-SIRT1 pathway. Experimental and Therapeutic Medicine 9(5): 18191826. DOI: $10.3892 / \mathrm{etm} .2015 .2302$

Vyskot B, AND HoBza R. 2004. Gender in plants: sex chromosomes are emerging from the frog. Trends in Genetics 20(9): 432-438. DOI:10.1016/j.tig.2004.06.006

TÖrJéK O, Bucherna N, Kiss E, Homoki H, Finta-Korpelova Z, Bocsa I, Nagy I, and HeszKy LE. 2002. Novel malespecific molecular markers (MADC5, MADC6) in hemp. Euphytica 127: 209-218.

White MJD. 1973. Animal Cytology and Evolution. Cambridge University Press, Cambridge.

WingE O. 1929. On the nature of sex chromosomes in Humulus. Hereditas 12: 53-63.

Yakovin N, Divashuk M, Razumova O, Soloviev A, and Karlov G. 2014. Use of laser microdissection for the construction of Humulus japonicus Siebold et Zuccarini, 1846 (Cannabaceae) sex chromosome-specific DNA library and cytogenetics analysis. Comparative Cytogenetics, 8(4): 323-336. DOI: 10.3897/CompCytogen.v8i4.8473

Zhang YH, DiStilio VS, Rehman F, Avery A, Mulcahy D, and Kesseli R. 1998. Y chromosome specific markers and the evolution of dioecy in the genus Silene. Genome 41: 141-147. 